\title{
Development and evaluation of scaffold-based nanosponge formulation for controlled drug delivery of naproxen and ibuprofen
}

\author{
Qurat-ul-ain Shoaib1, Nasir Abbas ${ }^{1 \star}$, Muhammad Irfan², Amjad Hussain, \\ Muhammad Sohail Arshad ${ }^{3}$, Syed Zajif Hussain ${ }^{4}$, Sumera Latif ${ }^{1}$, Nadeem Irfan \\ Bukhari $^{1}$ \\ ${ }^{1}$ University College of Pharmacy, University of the Punjab, Lahore, ${ }^{2}$ Department of Pharmacy, GC University, Faisalabad, \\ ${ }^{3}$ Department of Pharmacy, Bahauddin Zakariya University, Multan, ${ }^{4}$ Deprtment of Chemistry and Chemical Engineering, Syed \\ Babar Ali School of Science and Engineering, Lahore University of Management Sciences (LUMS), Lahore, Pakistan
}

${ }^{\star}$ For correspondence: Email: nasirabbas77@gmail.com; Tel: +92 3317724909

Sent for review: 13 April 2018

Revised accepted: 23 July 2018

\begin{abstract}
Purpose: To develop and evaluate nanosponge (NS) based sustained release formulations of naproxen (NAP) and ibuprofen (IBU).

Method: Six formulations of each candidate drug were prepared by emulsion solvent diffusion method, using varying ratios of polymers, i.e., ethyl cellulose and polyvinyl alcohol. The prepared formulations were evaluated for various parameters including production yield, particle size, polydispersity index, actual drug content and entrapment efficiency. Morphological, structural and thermo-analytical evaluations were performed using various techniques. In vitro release studies were performed on selected formulations.

Results: Nanosponge (NS) formulations of naproxen and ibuprofen were successfully prepared by emulsion solvent diffusion method. The particle size of naproxen and ibuprofen nanosponge formulations ranged from 347.6 to $1358 \mathrm{~nm}$ and 248.7 to $327.6 \mathrm{~nm}$, respectively. Formulations with equal proportion of ethyl cellulose and drug resulted in nanosponges with the desired particle size. Production yield, actual drug content and entrapment efficiency was dependent on the ratio of ethyl cellulose and polyvinyl alcohol. Formulations with equal proportion showed least PDI values 10.09 for $N A P$ and 0.07 for IBU) and highest zeta potential (-27.2 $\mathrm{mV}$ for $N A P$ and $-28.2 \mathrm{mV}$ for IBU). Morphological, structural and thermo-analytical analysis confirmed the encapsulation of drugs in nanosponge cavities, and exhibited spherical and porous morphology. Nanosponge formulations gave a sustained release pattern, based on Higuchi model. Drug release mechanism was Fickian followed Korsmeyer-Peppas model, due probably to the porosity of the nanosponge.

Conclusion: Sustained release nanosponge formulations of naproxen and ibuprofen have successfully been prepared.
\end{abstract}

Keywords: Nanosponge, Naproxen, Ibuprofen, Emulsion solvent diffusion method, Sustained release

This is an Open Access article that uses a funding model which does not charge readers or their institutions for access and distributed under the terms of the Creative Commons Attribution License (http://creativecommons.org/licenses/by/4.0) and the Budapest Open Access Initiative (http://www.budapestopenaccessinitiative.org/read), which permit unrestricted use, distribution, and reproduction in any medium, provided the original work is properly credited.

Tropical Journal of Pharmaceutical Research is indexed by Science Citation Index (SciSearch), Scopus, International Pharmaceutical Abstract, Chemical Abstracts, Embase, Index Copernicus, EBSCO, African Index Medicus, JournalSeek, Journal Citation Reports/Science Edition, Directory of Open Access Journals (DOAJ), African Journal Online, Bioline International, Open-J-Gate and Pharmacy Abstracts 


\section{INTRODUCTION}

Osteoarthritis, commonly known as inflammation of joints is one of the most prevailing disease causing disability in geriatric patients. Management of this disease requires a long term oral therapy of non-steroidal anti-inflammatory drugs (NSAIDs) for achieving desired therapeutic effect [1]. However recurrent oral administration of these agents can cause various gastrointestinal (Gl) complications, which may lead to ulcers and bleeding from various $\mathrm{Gl}$ lesions. In addition large number of NSAIDs belong to low solubility class (class II) of the Biopharmaceutical Classification System (BCS) and therefore require higher and frequent dosing which results in increased frequency of adverse effects.

To counter these problems, different types of sustained release dosage forms have been proposed and developed. Various sustained release mechanisms such as diffusion, dissolution, osmotic pressure, $\mathrm{pH}$ and ion exchange resin-drug complexes can be employed to modulate the release of drug from the dosage form. However these systems are associated with several limitations, e.g. stability issues, toxicity due to dose dumping, gastric irritation, instability at acidic $\mathrm{pH}$, high cost and less dose adjustment [2]. To overcome these problems, focus has been drawn towards nano technological approaches, where release of drug can be controlled in more precise manner. It has brought about the development of new techniques in drug formulations one of which is the nanosponge (NS).

NS are one of the colloidal carriers (nano sized) that have been currently suggested for the effective delivery of drug. It is a novel approach which offers controlled drug delivery to the active site which contribute towards reduced side effects, increased stability, targeted delivery, taste masking and ease of formulation [3]. Nanosponges are three-dimensional network like structure. The polymer is combined with crosslinkers acting as small hooks to join various parts of the polymer chains together. The overall effect is the formation of spherical microscopic particles comprising of countless interconnecting cavities having ability to encapsulate a wide range of drug substances [4]. The porous nature of the outer surface of the sponge offers control on the release of drug from the dosage form. Drug candidates having certain characteristics e.g. molecular weight between 100-400, solubility less than $10 \mathrm{mg} / \mathrm{mL}$ and melting point less than $250{ }^{\circ} \mathrm{C}$ are consider ideal for such type of preparations [5].
In the current study, aim was to develop NS based sustained release formulations of ibuprofen (IBU) and naproxen (NAP). These drugs belong to the NSAIDs class of drugs indicated for the management of swerve pain and inflammation associated with osteoarthritis. Both drugs have ideal characteristics required for NS preparation and can be considered as model drug for such type of study. Different formulations of NS were prepared by solvent emulsion diffusion method using ethyl cellulose (EC) and polyvinyl alcohol (PVA) as polymer and surfactant respectively. Prepared formulations were evaluated for physicochemical parameters including drug loading, entrapment efficiency and percentage yield. Structural characterization was done by different techniques including SEM, PXRD and FTIR. In-vitro bioavailability studies were also performed on the selected formulations.

\section{EXPERIMENTAL}

\section{Materials}

NAP, IBU, EC, PVA, dichloromethane (DCM) were purchased from Sigma Aldrich, Lahore, Pakistan. All the other chemicals were used without further purification and of analytical grade.

\section{Preparation of nanosponge formulations}

Six nanosponge (NS) formulations for each candidate drug (coded N1- N6 for NAP and I1-I6 for IBU) were prepared using the emulsion solvent diffusion method [6]. Two different concentrations of polyvinyl alcohol $(0.5$ and 0.75 $\% \mathrm{w} / \mathrm{v}$ ) were used with different ratios of ethyl cellulose and the candidate drugs as described in the Table 1.

The dispersed Phase was prepared by dissolving $E C$ and the drug in DCM $(20 \mathrm{~mL})$ and sonicated for ten minutes. Definite amount of PVA was dissolved by continuous stirring on water bath for 30 minutes at $80{ }^{\circ} \mathrm{C}$ in $150 \mathrm{~mL}$ of water to prepare the continuous phase. Dispersed phase was then added drop wise to the continuous phase. Mixture was stirred at $1000 \mathrm{rpm}$ for 2 hours. The formed solid was then collected by using membrane filter (pore size $0.45 \mu \mathrm{m}$ ) and washed with distilled water. Sample was dried for $2 \mathrm{~h}$ in an oven at $40{ }^{\circ} \mathrm{C}$ and for the removal of residual solvent it was kept in desiccator for $48 \mathrm{~h}$. The product was then packed and stored in airtight vials for characterization. 
Table 1: Composition of naproxen and ibuprofen nanosponges

\begin{tabular}{lcccccc}
\hline Formulation & N1 / I1 & N2 / I2 & N3 / I3 & N4 / I4 & N5 / I5 & N6 / I6 \\
\hline Drug content (g) & 2 & 2 & 2 & 2 & 2 & 2 \\
Polymer (EC) content (g) & 1 & 2 & 3 & 1 & 2 & 3 \\
Drug : polymer & $2: 1$ & $1: 1$ & $: 1.5$ & $2: 1$ & $1: 1$ & $: 1.5$ \\
PVA content (\%w/v) & 0.5 & 0.5 & 0.5 & 0.75 & 0.75 & 0.75 \\
Dichloromethane (mL) & 20 & 20 & 20 & 20 & 20 & 20 \\
\hline
\end{tabular}

\section{Drug content analysis}

For the quantitative determination validated spectrophotometric methods were used for both drugs. Stock solutions $(100 \mu \mathrm{g} / \mathrm{mL})$ of NAP and IBU were prepared by using phosphate buffer as a solvent with $\mathrm{pH} 7.4$ and 7.2 , respectively. Calibration curve of NAP was constructed using standard solutions with concentration range from $5-100 \mu \mathrm{g} / \mathrm{mL}$. Absorbance was measured at lambda max $\left(\lambda_{\max }\right)$ of NAP at $330 \mathrm{~nm}$ and concentration was determined by using a calibration curve with $R^{2}$ value of 0.993 . Calibration curve of Ibuprofen was constructed using standard solutions of concentration range from 5-30 $\mu \mathrm{g} / \mathrm{mL}$. Absorbance was measured at lambda max $\left(\lambda_{\max }\right)$ of IBU at $222 \mathrm{~nm}$. Concentration was measured by using calibration curve with $R^{2}$ value of 0.983 .

\section{Characterization of NS formulations}

The prepared formulations were initially characterized by production yield (\%), entrapment efficacy (\%), actual drug content (\%), particle size (PS), polydispersity index (PDI), zeta potential (ZP) and Fourier Transform Infrared Spectroscopy (FTIR). On the basis of initial parameters, best formulation was selected for further structural analysis by powder $\mathrm{x}$-ray diffraction (PXPD) and thermo-analytical methods such as differential scanning calorimetry (DSC) and thermal gravimetric analysis (TGA). Scanning electron microscopy (SEM) was used to investigate the morphology of prepared NS.

\section{Determination of production yield}

Production yield (PY) was calculated as in Eq 1.

PY $(\%)=($ WNS/WRM $) 100$

where WNS = weight of nanosponge obtained, WRM = weight of raw material (polymer + drug).

\section{Actual drug content and entrapment efficiency}

To calculate actual drug content (ADC) \%, nanosponge samples $(20 \mathrm{mg})$ of NAP and IBU were dissolved under sonication in $100 \mathrm{~mL}$ of phosphate buffer with $\mathrm{pH} 7.4$ and 7.2 respectively. Drug contents were determined spectrophotometrically (UV 2550 Shimadzu) by using the above described calibration curves. The following formulae were used to find the percentage actual drug content and entrapment efficiency (EE) \% of the prepared samples.

$\operatorname{ADC}(\%)=($ WDNS $/$ WNS $) 100$

where WDNS = actual weight of drug in nanosponges, WNS = weight of nanosponges obtained.

$E E(\%)=($ WDNS $/$ WD $) 100$

where WDNS = actual weight of drug in nanosponges, $W D=$ total weight of drug used.

Determination of particle size (PS), zeta potential (ZP) and polydispersity index (PDI)

Particle size (PS), polydispersity index (PDI) and zeta potential (ZP) of the nanosponge formulations were determined by zeta sizer (Malvern, ZSP Nano) [7]. For this, aqueous dispersions of nanosponges were diluted to an appropriate scattering intensity at $25^{\circ} \mathrm{C}$ and the average hydrodynamic diameter was measured with dynamic light scattering measurements using zeta sizer.

\section{Fourier transform infrared spectroscopy (FTIR):}

FTIR analysis of the pure drug, its physical mixture with polymer and nanosponge formulations was carried out by ATR-FTIR (Bruker, Alpha-P). The spectrum was scanned from 4000 to $400 \mathrm{~cm}^{-1}$.

For further evaluation, one formulation of each candidate drug was selected on the basis of the particle size, entrapment efficiency and FTIR analysis. For particle size, formulation with appropriate size of nanosponge, i.e. with an average diameter $<500 \mathrm{~nm}$ is selected, formulation with best entrapment efficiency and intact FTIR spectra for all the functional groups of candidate drug showing no interaction. 


\section{Morphology studies}

For the morphology studies of selected nanosponge formulation, Scanning Electron Microscopy (SEM) was used [8]. Particles of nanosponges were fixed on aluminum stub and coated with gold for improved contrast, using Sputter Coater (Denton, Desk V HP) operating at $40 \mathrm{~mA}$ for 25 seconds under vacuum. The morphology of particles was determined using Field Emission Scanning Electron Microscope (FE-SEM, Nova Nano SEM 450) operating at 15 $\mathrm{kV}$.

\section{Thermo-analytical studies}

The methods used to investigate the thermal characteristics of substances are Differential Scanning Calorimetry (DSC) and Thermal Gravimetric Analysis (TGA) [9]. Thermal behavior of the pure drug, its physical mixture with polymer and selected nanosponge formulation was determined using differential scanning calorimeter (TA instruments, SDT Q600) by heating from room temperature to $300^{\circ} \mathrm{C}$ under $\mathrm{N}_{2}$, providing heat at the rate of $10^{\circ} \mathrm{C} / \mathrm{min}$.

\section{Powder x-ray diffraction analysis (PXRD) studies}

Drug polymer complexation was analyzed by $X$ ray diffraction [10]. X-ray diffraction analysis of the pure drug, its physical mixture with polymer and selected formulation was carried out in an $\mathrm{x}$ ray diffractometer (D8 Advance, Bruker) with $\mathrm{Cu}$ $\mathrm{Ka}$ radiation $\left(\lambda=1.54060 \mathrm{~A}^{\circ}\right)$. The rate of scanning was $100 / \mathrm{min}$ and diffraction angle $2 \Theta$ was $10-800$.

\section{In-vitro release studies}

In-vitro drug release studies of pure drug, its physical mixture and the selected formulations were performed in a Type II dissolution test apparatus (ErwekaDT700), at $37 \pm 0.5^{\circ} \mathrm{C}$ and at a paddle speed of $50 \mathrm{rpm}$. Sample of nanosponge formulation equivalent to $100 \mathrm{mg}$ of the pure candidate drug was used for the analysis [11]. Dissolution medium $(900 \mathrm{~mL})$ of $\mathrm{pH}$ 7.4 for naproxen and $\mathrm{pH} 7.2$ for lbuprofen for 12 $\mathrm{h}$ was used. Samples $(5 \mathrm{~mL})$ were withdrawn from the dissolution apparatus at different time intervals (15, 60 and $120 \mathrm{~min}$ ) and then filtered. The withdrawn samples were replenished with 5 $\mathrm{mL}$ of fresh dissolution media to maintain the sink conditions. The drug content was determined at $\lambda_{\max } 330 \mathrm{~nm}$ for naproxen and at $\lambda_{\max } 222 \mathrm{~nm}$ for lbuprofen.

\section{Kinetic analysis of drug release}

To study the drug release mechanism of nanosponge formulations, the release data of selected nanosponge formulations was fitted to zero-order, first-order, Higuchi, KorsmeyerPeppas, Hixson- Crowell and Baker Lonsdale kinetic models. The kinetic model with the highest coefficient of correlation $\left(R^{2}\right)$ value was considered to be the best fit model for describing the release of drug from the nanosponges.

\section{RESULTS}

In order to assess the effect of polymers on the properties of nanosponges, twelve formulations ( 6 for each candidate drug) with different ratios of drug, EC and PVA (as shown in Table 1) were prepared. For NAP, all formulations resulted in the formation of nanosponges, while in case of IBU; nanosponges were successfully formed only with formulations (I1 and I2) having low proportions of EC and PVA. All the other formulations (I3 to 16 ) resulted in a flexible plastic-like sticky paste shown in Figure 1.

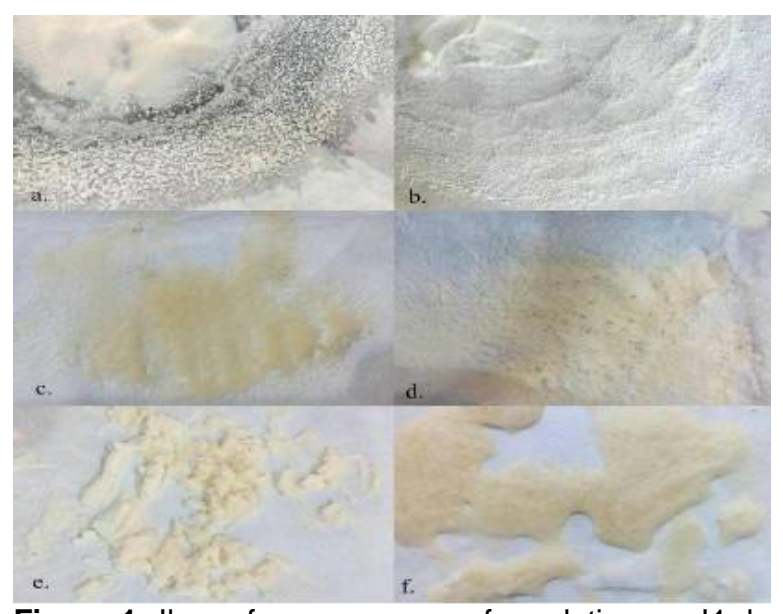

Figure 1: Ibuprofen nanosponge formulations a. I1, b. 12, c. 13 , d. 14 , e. 15 , f. 16

Production yield of NAP formulations (N1-N6) was in the range of 70 to $86.6 \%$ as shown in Table 2. The value of this parameter for IBU formulations 11 and 12 was 80 and $75 \%$, respectively.

It was observed that the actual drug content and entrapment efficiency of the nanosponge formulations of both drugs are dependent on the Drug: EC ratio. Maximum values of these parameters were achieved with equal proportions of drug and EC (N2 for Naproxen and 12 for Ibuprofen). With respect to PVA, entrapment efficiency decreased with increase in PVA concentration from 0.5 to $0.75 \%$. 
Table 2: Characteristics of nanosponge formulations

\begin{tabular}{|c|c|c|c|c|c|c|c|}
\hline $\begin{array}{l}\text { Formulation } \\
\text { code }\end{array}$ & $\begin{array}{l}\text { Nanosponge } \\
\text { Formation }\end{array}$ & $\begin{array}{c}\text { Production } \\
\text { yield (\%) }\end{array}$ & $\begin{array}{c}\text { Actual } \\
\text { drug } \\
\text { content } \\
(\%)\end{array}$ & $\begin{array}{c}\text { Entrapment } \\
\text { efficiency } \\
(\%)\end{array}$ & $\begin{array}{l}\text { Zeta } \\
\text { Size } \\
(\mathrm{nm})\end{array}$ & PDI & $\begin{array}{c}\text { Zeta } \\
\text { potential } \\
(\mathrm{mV})\end{array}$ \\
\hline N1 & + & 86.66 & 57.3 & 74.5 & 347.6 & 0.266 & -19.7 \\
\hline N2 & + & 84.5 & 49.4 & 83.5 & 464.7 & 0.099 & -27.2 \\
\hline N3 & + & 80 & 33.25 & 66.5 & 630.8 & 0.558 & -17.3 \\
\hline N4 & + & 83.33 & 54 & 67.5 & 695.1 & 0.286 & -21.4 \\
\hline N5 & + & 75 & 49.33 & 74 & 993.6 & 0.851 & -25.2 \\
\hline N6 & + & 70 & 29.45 & 51.55 & 1358.0 & 1 & -10.7 \\
\hline I1 & + & 80 & 55.7 & 66.84 & 248.7 & 0.263 & -21.8 \\
\hline 12 & + & 75 & 58.4 & 87.6 & 327.6 & 0.076 & -28.2 \\
\hline 13 & - & & & & & & \\
\hline 14 & - & & & & & & \\
\hline 15 & - & & & & & & \\
\hline 16 & - & & & & & & \\
\hline
\end{tabular}

Mean particle size, PDI and zeta potential values of prepared NS formulations are given in Table 2 . The mean particle size was dependent on the quantity of polymers. Increasing the polymer fraction significantly increased the particle size. Particle size was also found to be directly proportional to PVA concentrations, whereby smaller particle size was achieved with $0.5 \%$ concentration while higher concentration $(0.75$ $\%$ resulted in a larger particle size. Polydispersity Index (PDI) is a measure of the range of size distribution. Values greater than 1 indicate that the distribution is poly-dispersed. N2 and 12 formulations (with equal proportion of drug and polymer) had the least PDI value i.e. 0.09 and 0.07 respectively. This shows that all the particles of these formulations are in monodispersed region i.e. are of similar size. Electric charge on a nanoparticle surface is measured by zeta potential. Higher magnitude of zeta potential shows increased stability due to a greater electrostatic repulsion among the particles. Our results have shown that the formulations (N2 and 12 with equal proportion of drug and polymer) with least PDI values have the highest Zeta potential suggesting stable formulations.

\section{FTIR analysis}

FTIR spectra of the pure drug, its physical mixture and nanosponge formulations are compared in Figure 2 and 3 for NAP and IBU respectively. The spectrum of NAP showed three characteristics peaks at 3151.50, 2973.60 and $2938.19 \mathrm{~cm}^{-1}$ in functional group region. These peaks correspond to the stretching of $\mathrm{O}-\mathrm{H}$ and $\mathrm{C}$ $\mathrm{H}$ of carboxylic group. Apart from the characteristic peaks in the functional region, peaks specific to NAP were also present in the fingerprint region of the spectrum. In the physical mixture all the peaks remained intact and no significant shifts were observed. This represents lack of chemical interaction between the drug and the polymers. In nanosponge formulations (N1-N6), there is a broadening and disappearance of the drug peaks, which shows that the drug has been encapsulated.

FTIR spectra of pure IBU in Figure 4 showed characteristic peaks at 1725.15 and $2874.21 \mathrm{~cm}^{-}$ 1 due to carbonyl and hydroxyl stretching respectively. $\mathrm{C}=\mathrm{C}$ bending of the aromatic ring present at $1684.4 \mathrm{~cm}^{-1}$ and the $\mathrm{C}-\mathrm{H}$ stretch present at $2973.24 \mathrm{~cm}^{-1}$. Medium band at $600-$ $900 \mathrm{~cm}^{-1}$ corresponds to the $\mathrm{C}-\mathrm{H}$ bending and ring puckering. These Characteristic peaks were also found intact in spectra of the physical mixture and nanosponge formulations (I1-I2). Broadening and disappearance of the drug peaks suggests that the drug has been successfully encapsulated in the NS core.

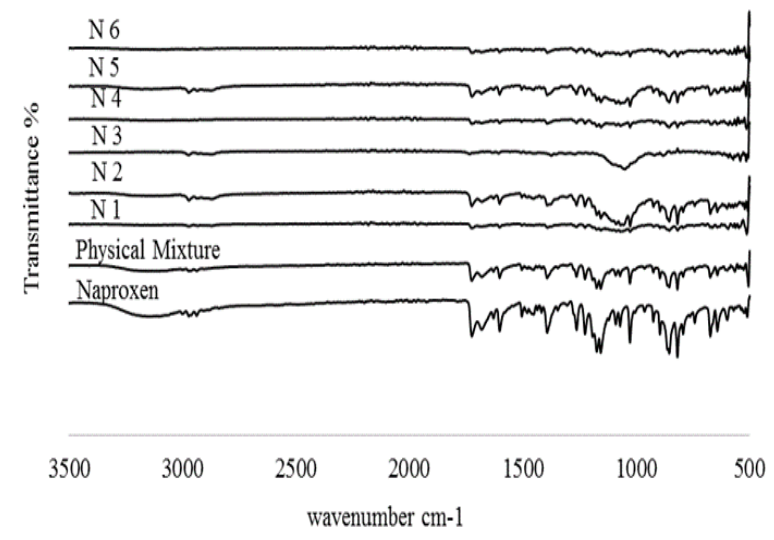

Figure 2: FTIR spectra of Naproxen, Physical Mixture and Naproxen nanosponge formulations (N1-N6)

\section{Morphological, structural and thermoanaly- tical characteristics}

Morphological, Structural and Thermoanalytical characterization of selected formulations (N2 and 12) were performed by SEM, PXRD, DSC and TGA techniques. These formulations were 
selected on the basis of particle size, entrapment efficiency and FTIR analysis. As the size of N2 and 12 formulation was 464.7 and $327.6 \mathrm{~nm}$ respectively, which is considered appropiate for nanosponge formulation. Entrapment efficacy of $\mathrm{N} 2$ and 12 formulations were 83.5 and $87.6 \%$ respectively, which is the highest among all other prepared formulations. FTIR spectra was also intact showing no chemical interaction.

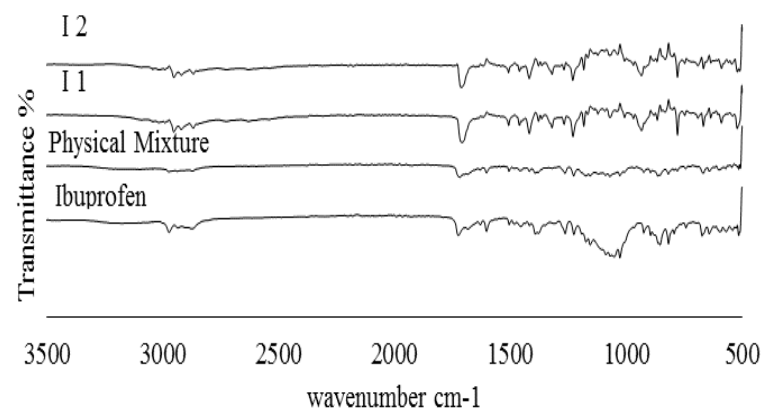

Figure 3: FTIR spectra of ibuprofen, physical mixture and ibuprofen nanosponge formulations (I1- I2)

\section{Scanning electron microscopy (SEM)}

SEM examination of formulations $\mathrm{N} 2$ and $\mathrm{I} 2$ showed porous, spherical and nanosized particles. Figure 4 and Figure 5 clearly depicts the porous and spongy texture of the nanosponges.

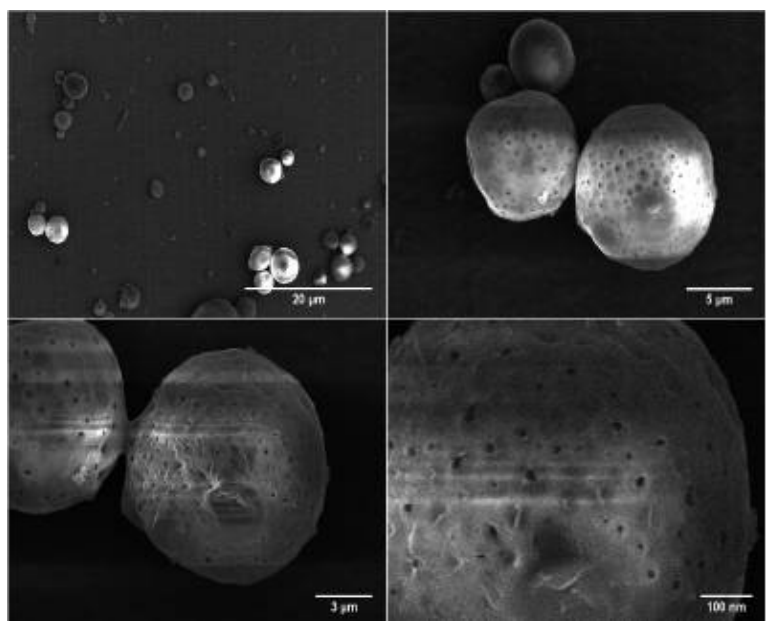

Figure 4: SEM images of Naproxen nanosponge formulation (N2)

\section{Thermo-analytical analysis}

Differential scanning calorimetry (DSC) thermogram of pure NAP, its physical mixture with polymer and nanosponge formulation (N2) is shown in Figure 6a. DSC curve of pure Naproxen showed an endothermic peak at $154.8^{\circ} \mathrm{C}$ corresponding to its melting point [12]. DSC curve of physical mixture showed same thermogram as that of the pure drug, indicating the lack of chemical interactions between the drug and the polymer. DSC curve of the nanosponge formulation showed that the drug did not melt as no peak was observed at its melting temperature.

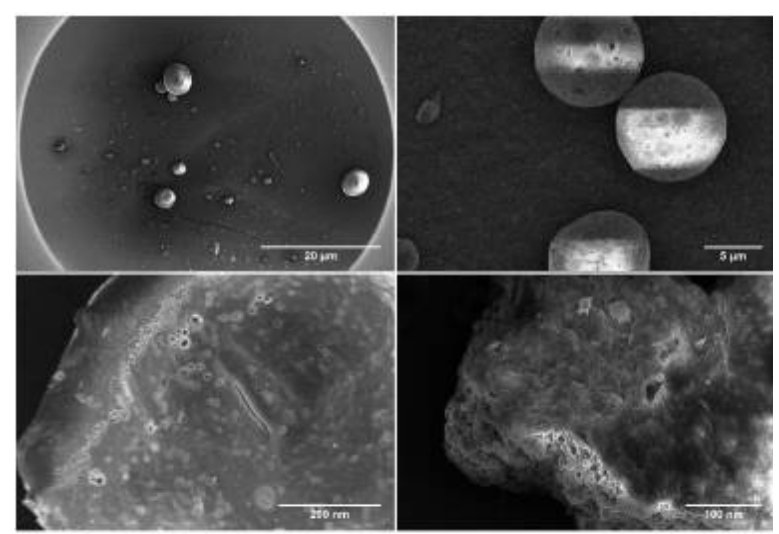

Figure 5: SEM images of ibuprofen nanosponge formulation (I2)

Figure 6c shows a comparison of TGA curve of pure drug, its physical mixture and nanosponge formulation. Pure drug NAP shows thermal stability up to $\sim 180^{\circ} \mathrm{C}$. A sharp decline after this point represents the fast decomposition of the samples. TGA Curve of physical mixture shows almost the same thermal behavior as that of the pure drug. However TGA curve of NAP nanosponges shows a shallow decline after 250 ${ }^{\circ} \mathrm{C}$ with minimum $\%$ weight loss, indicating better stability of this formulation.

Two non-symmetrical endothermic peaks were observed in the DSC curve of pure IBU at 76.4 and $230^{\circ} \mathrm{C}$ (Figure $6 \mathrm{~b}$ ) demonstrating the melting point and decomposition of the sample, respectively [13]. Similar DSC curve was observed for physical mixture, suggesting a lack of chemical interaction between the drug and the polymer. DSC curve of NS formulation shows the absence of drug melting peak indicating the successful encapsulation of drug within the nanosponge cavities.

TGA analysis of pure IBU, its physical mixture and IBU nanosponge formulation is compared in Figure 6d. TGA curve of pure drug and physical mixture show a sharp decline starting from $150^{\circ} \mathrm{C}$, manifesting the decomposition of the samples. In contrast, the TGA of nanosponge formulation shows a straight line up to the studied temperature, showing their thermal stability up to $300{ }^{\circ} \mathrm{C}$. 


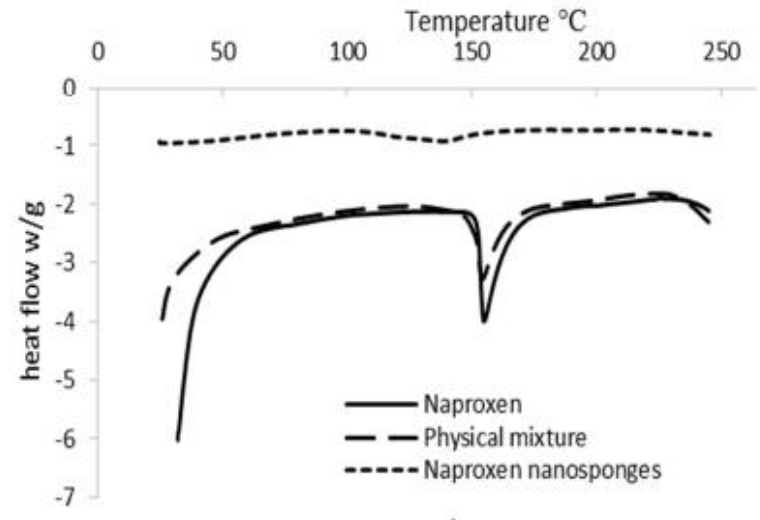

a)

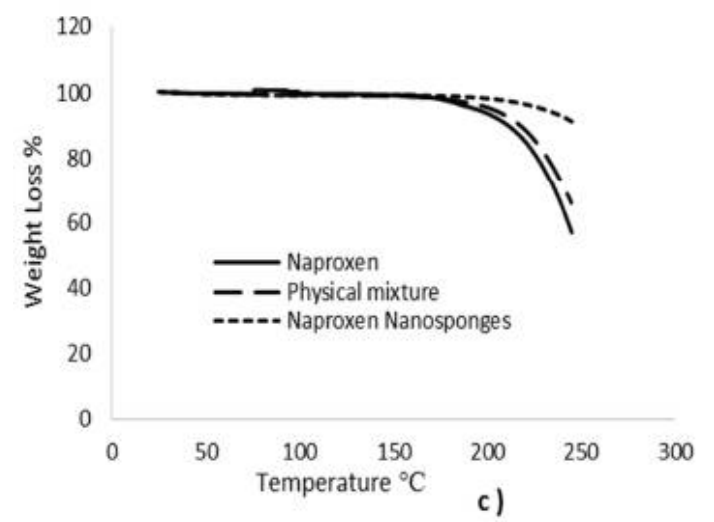

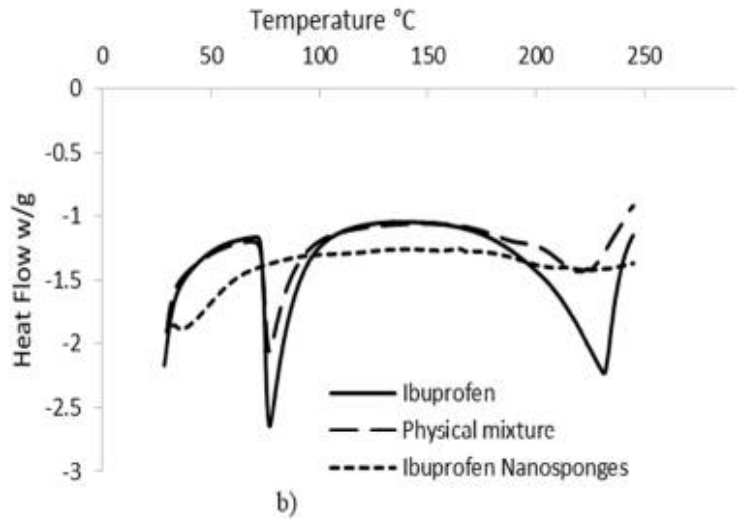

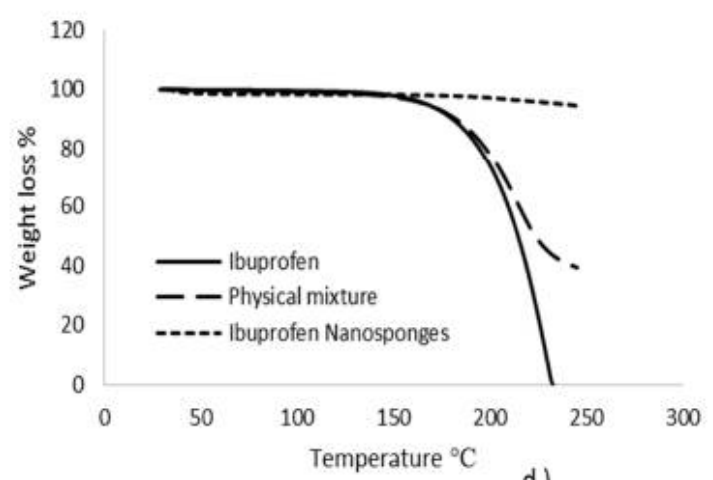

d)

Figure 6: a) DSC of Naproxen, Physical Mixture and naproxen nanosponge formulation N2. b) DSC of Ibuprofen, Physical Mixture and Ibuprofen nanosponge formulation I2. c) TGA of Naproxen, Physical mixture and naproxen nanosponge formulation N2. d) TGA of Ibuprofen, Physical mixture and Ibuprofen nanosponge formulation I2.

\section{Powder x-ray diffraction (PXRD)}

Powder X-ray Diffraction (PXRD) patterns of pure drug, its physical mixture and nanosponge formulations of NAP and IBU are shown in Figure $7 \mathrm{a}$ and $7 \mathrm{~b}$, respectively. The diffraction patterns of pure drugs show sharp and intense peaks indicating the crystalline nature of the sample. These peaks can also be observed in the physical mixtures showing lack of chemical or physical interactions between the drug and the polymer. PXRD pattern obtained from nanosponge samples of NAP show masking of crystalline peaks which suggest the successful encapsulation of the drug within the nanosponge core. In case of IBU, the nanosponge formulation has shown a pattern of an amorphous nature, as no diffraction peaks were observed [14].

\section{In vitro release and kinetics}

The in vitro release study of the pure drug, its physical mixture and selected nanosponge formulations i.e. N2 and 12 was performed for 12 h. The percentage drug release for pure drugs was comparable with their respective physical mixtures. The percentage drug release was
$95.78 \%$ in $12 \mathrm{~h}$ for $\mathrm{N} 2$ and $81.75 \%$ in $7.25 \mathrm{~h}$ for 12 formulation as shown in Figure 8.
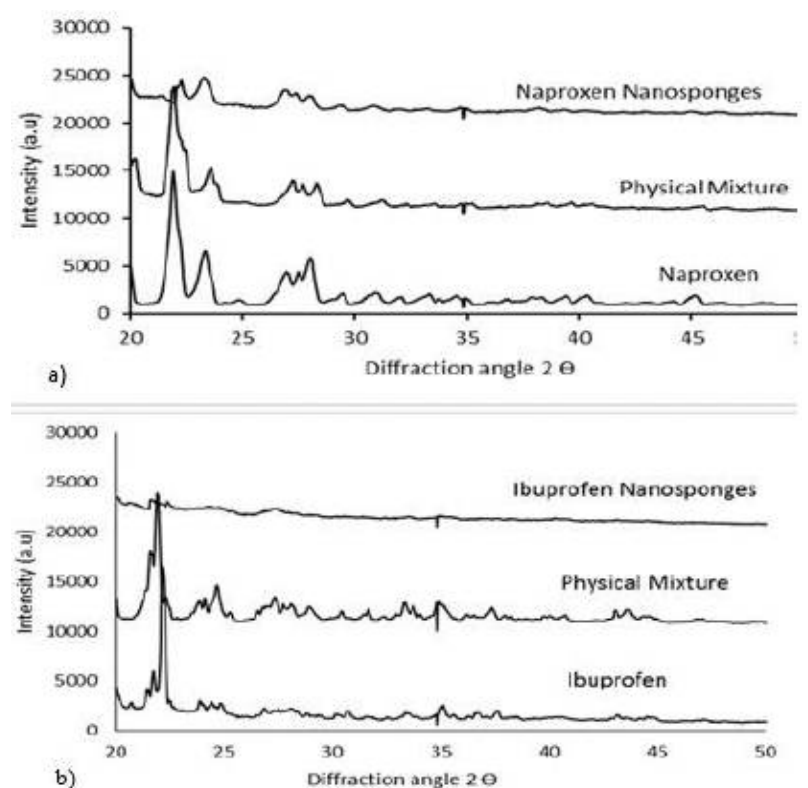

Figure 7: a) XRD of Naproxen, Physical Mixture, Naproxen Nanosponges (N2), b) XRD of Ibuprofen, Physical Mixture, Ibuprofen Nanosponges (I2) 

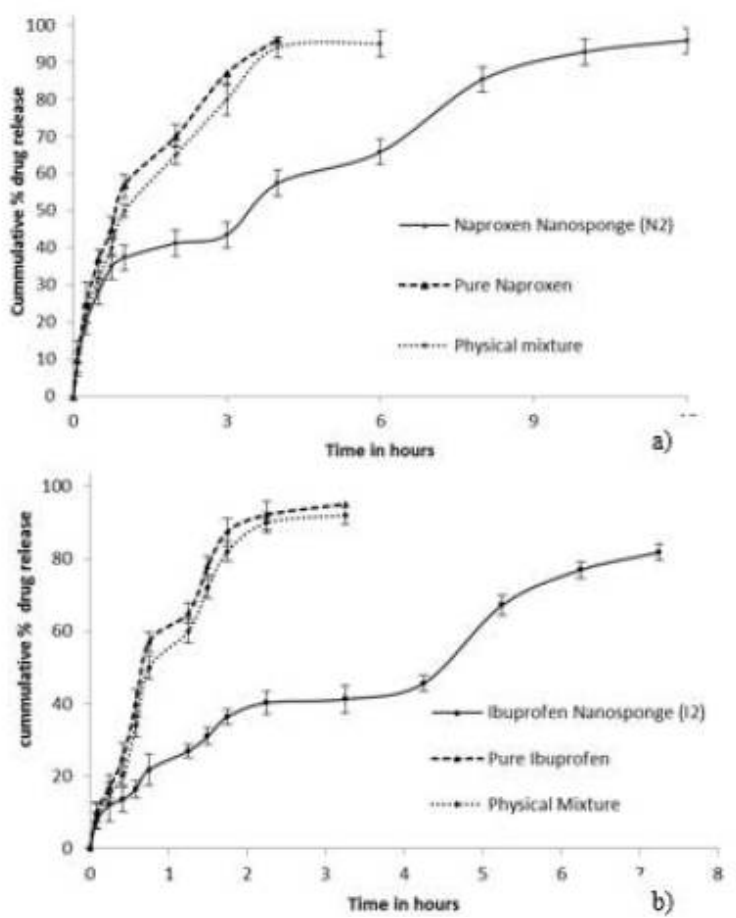

Figure 8: Graph of in vitro cumulative drug release versus time (hours) for (a) Naproxen nanosponge (N2) and (b) Ibuprofen nanosponge (I2)

Various kinetic models such as Zero Order Kinetics, First Order Kinetics, Higuchi plot, Korsmeyer-Peppas plot, Hixon Crowell plot and Baker Lonsdale plot were applied to dissolution data obtained from the NS formulations. The interpretation of data of both drugs showed that maximum linearity (highest $R^{2}$ value) was found with Higuchi classical model of diffusion; $\left(\mathrm{R}^{2}=\right.$ 0.9597 and 0.9453 for NAP and IBU respectively) as shown in Table 3. This manifests that the drug release mechanism is by diffusion process. To determine whether this diffusion process is Fickian or non Fickian, the Korsmeyer-Peppas model was applied to $60 \%$ of the release data. The results obtained after the application of Korsmeyer-Peppas model showed ' $n$ ' values to be less than 0.5 which suggests that the diffusion process is obeying Fick's law of diffusion [15].

\section{DISCUSSION}

The emulsion solvent diffusion method with varying ratios of pure drug and polymer was used to successfully prepare the Naproxen nanosponge formulations (N1-N6). However two formulations of Ibuprofen (I1 and I2) out of the six were obtained while the rest resulted in a flexible plastic-like sticky paste. This may be due to the effect of IBU on the plasticity of EC and PVA as IBU is reported as a non-traditional plasticizer of ethyl cellulose [16]. The efficiency of the plasticizer relies upon concentration and level of interaction between the polymer and the plasticizer [17]. With the rise in concentration of the polymer in the formulations 13 to 16 , the plastic behavior of the mixture became more evident and therefore the sponges were unable to retain their shape and were consequently converted into flexible mass [18].

Table 3: Release kinetics of N2 and 12 nanosponge formulations

\begin{tabular}{lll}
\hline Kinetic model & Ibuprofen & Naproxen \\
\hline zero -order & $\mathrm{R}^{2}=0.839$ & $\mathrm{R}^{2}=0.553$ \\
& $\mathrm{~K}_{0}=0.209$ & $\mathrm{~K}_{0}=0.164$ \\
First order & $\mathrm{R}^{2}=0.929$ & $\mathrm{R}^{2}=0.852$ \\
& $\mathrm{~K}_{1}=0.004$ & $\mathrm{~K}_{1}=0.004$ \\
Higuchi & $\mathrm{R}^{2}=0.945$ & $\mathrm{R}^{2}=0.959$ \\
Korsmeyer Peppas & $\mathrm{K}_{\mathrm{H}}=3.528$ & $\mathrm{~K}_{\mathrm{H}}=3.734$ \\
& $\mathrm{R}^{2}=0.962$ & $\mathrm{R}^{2}=0.929$ \\
& $\mathrm{KKP}=3.563$ & $\mathrm{KKP}=8.22$ \\
Hixson Crowell & $\mathrm{n}=0.471$ & $\mathrm{n}=0.345$ \\
& $\mathrm{R}^{2}=0.915$ & $\mathrm{R}^{2}=0.814$ \\
Baker Lonsdale & $\mathrm{KHC}=0.001$ & $\mathrm{KHC}=0.001$ \\
& $\mathrm{R}^{2}=0.905$ & $\mathrm{R}^{2}=0.956$ \\
& $\mathrm{KBL}=0.000$ & $\mathrm{KBL}=0.000$ \\
\hline
\end{tabular}

It was observed that the production yield is inversely proportional to the concentration of both the polymers. Increasing the concentration of EC and PVA decreased the production yield. This may be due to the following reasons: First, larger amounts of polymer increased the wall thickness of the nanosponge (as indicated by a larger particle size). Second, a higher concentration of PVA produced hydrophobic regions, due to which some of the drug and polymer was dissolved [19]. Furthermore, increased foaming was witnessed during the nanosponge preparation with increase in the concentration of polymers hindering the formation of the nanosponges. This foaming consequently reduced the filtration rate thereby increasing the synthesis time.

It was noted that when the quantity of EC was more than that of the drug, aggregate formation increased. This is primarily due to the increased viscosity of internal phase and results in decreased entrapment of drug [20]. Entrapment efficiency also decreased with increase in PVA concentrations. This may be due to the fact that PVA acts as a nonionic emulsifier. It might also be possible that at higher concentration the drug molecules associate away from interface making an alternative hydrophobic region, due to which some portions of the drug was dissolved and subsequently decrease the entrapment efficiency [21].

The results also illustrated that the mean particle size had a direct relation with the amount of polymer used in the preparation. Increasing the 
polymers (EC and PVA) fraction significantly increased the particle size due to foaming and aggregation. Larger quantities of EC increased the viscosity of the system which created hindrances in the formation of smaller droplets $[20,22]$. At lower concentrations, diffusion of the internal phase (dichloromethane) into the external phase (aqueous phase) improved, reducing the time for droplet formation which resulted in smaller particle sizes. Also, it was reported that surfactants (PVA) at higher concentration (above critical micellar concentration) may not contribute to decrease in particle size [23]. Increased amount of PVA also increases the viscosity of the medium thus reducing the sheer stress, which is an important variable for decreasing the particle size. Moreover, PVA sticks to the surface of nanosponges and remain bound to them after repetitive washings which increases the size of the particles [24].

Structural analysis by FTIR of nanosponge formulations of both candidate drugs showed broadening and disappearance of drug peaks which suggests complex formation, and thus drug encapsulation. Morphological analysis by SEM confirmed porous morphology and that the pores in the nanosponge formulations were channeled inwards. This may be due to the impressions of the solvent (DCM) diffusion on nanosponge surface [6].

Thermo-Analytical studies (DSC and TGA) revealed that the drug is well incorporated into the nanosponge core and therefore nanosponges can be processed at a relatively higher temperature and impart stability on the formulation as compared to the pure drug. The thermal behavior of nanosponges indicated that the polymers hindered the re-crystallization of the drug possibly due to the drug's encapsulation in nanosponge cavities. The degree of crystallinity of both drugs was significantly reduced in the nanosponge formulations as compared to its physical mixture. This indicates that the drug was able to disperse almost homogenously, as a solid solution or as a metastable molecular dispersion.

PXRD results suggest that the drug loading in the nanosponge formulations is not due to mechanical mixing of the components but due to the encapsulation of the drug in the nanosponge cavities. The encapsulated drug may be in amorphous or solubilized form within the nanosponge channels [25].

In vitro release of Naproxen and Ibuprofen nanosponge formulations (N2 and 12) showed sustained release pattern and revealed that the drug release mechanism is by diffusion which may be controlled by the porosity of nanosponges.

\section{CONCLUSION}

Nanosponge formulations of naproxen and ibuprofen with particle size in the nano range, based on ethyl cellulose and polyvinyl alcohol as polymers, have been successfully prepared by emulsion solvent diffusion method. Physicochemical parameters of the prepared nanosponge formulations including yield, drug loading, entrapment efficiency and particle size are dependent upon the polymer concentrations. The nanosponges consist of spherical spongy structures in which the drug is encapsulated. Mode of drug release follows a sustained pattern by Fickian diffusion.

\section{DECLARATIONS}

\section{Acknowledgement}

This work was funded by Higher Education Commission, Pakistan.

\section{Conflict of interest}

The authors declare that no conflict of interest is associated with this work.

\section{Contribution of authors}

We declare that this work was done by the authors named in this article and all liabilities pertaining to claims relating to the content of this article will be borne by them. Qurat-ul-ain shoaib did the overall lab work and wrote the manuscript. Sumera Latif assisted in lab work. Syed Zajif Hussain assisted in characterization of nanosponges. Nasir Abbas and Nadeem Irfan Bukhari supervised the work and reviewed the manuscript. Sohail Arshad and Amjad Hussain did the technical review.

\section{REFERENCES}

1. Crofford LJ. Use of NSAIDs in treating patients with arthritis. Arthritis Res Ther 2013; 15(3): S2.

2. Karna $S$, Chaturvedi $S$, Agrawal VM. Formulation approaches for sustained release dosage forms: a review. Asian J Pharm Clin Res 2015; 8(5): 46-53.

3. Ahmed RZ, Patil G, Zaheer $Z$. Nanosponges-a completely new nano-horizon: pharmaceutical applications and recent advances. Drug Dev Ind Pharm 2013; 39(9): 1263-1272. 
4. Selvamuthukumar S, Anandam S, Krishnamoorthy K, Rajappan M. Nanosponges: A novel class of drug delivery system-Review. J Pharm Pharm Sci 2012; 15(1): 103-111.

5. Szejtli J. Cyclodextrin complexed generic drugs are generally not bio-equivalent with the reference products: therefore the increase in number of marketed drug/cyclodextrin formulations is so slow. $\mathrm{J}$ Incl Phenom Macrocycl Chem 2005; 52(1-2): 1-11.

6. Sharma R, Pathak K. Polymeric nanosponges as an alternative carrier for improved retention of econazole nitrate onto the skin through topical hydrogel formulation. Pharm Dev Technol 2011; 16(4): 367-376.

7. Jilsha G, Viswanad V. Nanosponges: A Novel Approach of Drug Delivery System. Int J Pharm Sci Rev Res 2013; 19(2): 119-123.

8. Swaminathan $S$, Pastero L, Serpe L, Trotta F, Vavia $P$, Aquilano D, Trotta M, Zara G, Cavalli R. Cyclodextrinbased nanosponges encapsulating camptothecin: physicochemical characterization, stability and cytotoxicity. Eur J Pharm Biopharm 2010; 74(2): 193201.

9. Tejashri G, Amrita B, Darshana J. Cyclodextrin based nanosponges for pharmaceutical use: $A$ review. Acta pharm 2013; 63(3): 335-358.

10. Shringirishi M, Prajapati SK, Mahor A, Alok S, Yadav P, Verma A. Nanosponges: a potential nanocarrier for novel drug delivery-a review. Asian Pac J Trop Dis 2014; 4: S519-S526.

11. Subhash PB, Mohite SK. Formulation design \& development of Artesunate Nanosponge. Eur J Pharm Med Res 2016; 3(5): 206-211.

12. Mello VA, Ricci-Júnior E. Encapsulation of naproxen in nanostructured system: structural characterization and in vitro release studies. Quím Nova 2011; 34(6): 933939.

13. Tiţa B, Fuliaş A, Szabadai Z, Rusu G, Bandur G, Tiţa D. Compatibility study between ibuprofen and excipients in their physical mixtures. J Therm Anal Calorim 2010; 105(2): 517-527.

14. Cavalli R, Trotta F, Tumiatti Wander. Cyclodextrin-based nanosponges for drug delivery. J Incl Phenom Macrocycl Chem 2006; 56(1-2): 209-213.
15. Mathew ST, Devi SG, Sandhya KV. Formulation and evaluation of ketorolac tromethamine-loaded albumin microspheres for potential intramuscular administration. AAPS PharmSciTech 2007; 8(1): E100-E108.

16. De Brabander C, Van den Mooter G, Vervaet C, Remon $J P$. Characterization of ibuprofen as a nontraditional plasticizer of ethyl cellulose. J Pharm Sci 2002; 91(7): 1678-1685.

17. Wu C, McGinity JW. Non-traditional plasticization of polymeric films. Int J Pharm 1999; 177(1): 15-27.

18. Gutiérrez-Rocca J, McGinity JW. Influence of water soluble and insoluble plasticizers on the physical and mechanical properties of acrylic resin copolymers. Int $\mathrm{J}$ Pharm 1994; 103(3): 293-301.

19. Mayur K, Ramesh K, Nitin J, Prashant P, Rajendra G, Jeevan $N$. Ethyl cellulose based microsponge delivery system for antifungal vaginal gels of tioconazole. JDDT 2013; 3(6): 14-20.

20. Abdelmalak NS, El-Menshawe SF. A new topical fluconazole microsponge loaded hydrogel: Preparation and characterization. Int J Pharm Pharm Sci 2012; 4(1): 460-469.

21. Nokhodchi A, Jelvehgari $M$, Siahi MR, Mozafari MR. Factors affecting the morphology of benzoyl peroxide microsponges. Micron 2007; 38(8): 834-840.

22. Yang $Y$, Chung $T$, Ng Ngee P. Morphology, drug distribution, and in vitro release profiles of biodegradable polymeric microspheres containing protein fabricated by double-emulsion solvent extraction/evaporation method. Biomaterials 2001; 22(3): 231-241.

23. Li M, Rouaud O, Poncelet D. Microencapsulation by solvent evaporation: State of the art for process engineering approaches. Int J Pharm 2008; 363(1): 2639.

24. Narayanan K, Subrahmanyam VM, Venkata RJ. A fractional factorial design to study the effect of process variables on the preparation of hyaluronidase loaded plga nanoparticles. Enzyme Res 2014; 2014.

25. Torne S, Darandale S, Vavia P, Trotta F, Cavalli $R$. Cyclodextrin-based nanosponges: effective nanocarrier for Tamoxifen delivery. Pharm Dev Technol 2013; 18(3): 619-625. 